\title{
DT-13 attenuates human lung cancer metastasis via regulating NMIIA activity under hypoxia condition
}

\author{
XIAO-HUI WEI $^{1 *}$, SEN-SEN LIN ${ }^{2 *}$, YANG LIU ${ }^{1}$, REN-PING ZHAO ${ }^{2}$, GHULAM JILANY KHAN ${ }^{2}$, HONG-ZHI DU ${ }^{2}$, \\ TING-TING MAO ${ }^{1}$, BO-YANG YU ${ }^{3}$, RUI-MING LI ${ }^{4}$, SHENG-TAO YUAN ${ }^{1}$ and LI SUN ${ }^{2}$ \\ ${ }^{1}$ Jiangsu Center for Pharmacodynamics Research and Evaluation, ${ }^{2}$ Jiangsu Key Laboratory of Drug Screening, \\ ${ }^{3}$ State Key Laboratory of Natural Medicines, China Pharmaceutical University, Nanjing; ${ }^{4}$ Tasly Research Institute, \\ Tianjin Tasly Hodling Group Co., Ltd., Tianjin, P.R. China
}

Received January 24, 2016; Accepted March 8, 2016

DOI: $10.3892 /$ or.2016.4879

\begin{abstract}
Cancer metastasis plays a major role in tumor deterioration. Metastatic processes are known to be regulated by hypoxic microenvironment and non-muscle myosin IIA (NMIIA). DT-13, a bioactive saponin monomer isolated from Ophiopogon japonicus, has been reported to inhibit various cancer metastasis, but whether NMIIA is involved in the anti-metastatic activity of DT-13 under hypoxia remains to be determined. Thus, this study aims to clarify the role of DT-13 in regulating 95D cell metastasis under hypoxic microenvironment and to further investigate whether NMIIA is involved in the anti-metastatic mechanism of DT-13. We found that DT-13 significantly inhibited 95D cells metastasis in vitro and in vivo. Furthermore, hypoxia significantly inhibited the expression of NMIIA and redistributed NMIIA to the cell periphery, whereas DT-13 reversed the hypoxic effects by upregulating the expression of NMIIA. Moreover, DT-13 treatment redistributed NMIIA to the nuclear periphery and reduced the formation of F-actin in 95D cells. In addition, we found that the Raf-ERK1/2 signaling pathway is involved in regulation of NMIIA by DT-13. Collectively, these findings support NMIIA as a target of DT-13 to prevent lung cancer metastasis.
\end{abstract}

Correspondence to: Professor Sheng-Tao Yuan, Jiangsu Center for Pharmacodynamics Research and Evaluation, China Pharmaceutical University, 24 Tong JiaXiang St., Nanjing, Jiangsu 210009, P.R. China E-mail: cpuyuanst@163.com

Professor Li Sun, Jiangsu Key Laboratory of Drug Screening, China Pharmaceutical University, 24 Tong JiaXiang St., Nanjing, Jiangsu 210009, P.R. China

E-mail: cpusunli@126.com

*Contributed equally

Abbreviations: DT-13, saponin monomer of dwarf lilyturf tuber; NMIIA, non-muscle myosin IIA; F-actin, filamentous actin; ERK1/2, extracellular signal-regulated kinase1/2; FAs, focal adhesions

Key words: DT-13, NMIIA, metastasis, hypoxia, lung cancer, Raf-ERK1/2

\section{Introduction}

Pulmonary carcinoma is one of the most common types of cancer and the leading cause of cancer-related deaths. Approximately $90 \%$ of lung cancer deaths are the result of tumor metastasis rather than of primary tumor proliferation (1). Tumor metastasis is a multi-step process that requires the formation of new membrane extensions at the leading edge of the cell, the formation of new adhesions to the substrate and contractions at the trailing edge to move the cell body forward. An increasing number of studies have shown that the tumor microenvironment plays an important role in cancer progression, especially in tumor metastasis.

Hypoxia is one of the most critical features of tumor microenvironment, which is associated with stem cell maintenance (2), cell immortalization, glucose metabolism (3), epithelialmesenchyme transition (4), $\mathrm{pH}$ regulation (5), invasion and metastasis (6). The major regulator of hypoxic responses is hypoxia-inducible factor-1 (HIF-1), which accumulates at low oxygen levels and acts as a transcription factor for $>100$ target genes $(7,8)$. However, the molecular basis for the ability of cancer cells to migrate, invade and metastasize under hypoxic conditions is still unclear.

A critical component of cell migration is thought to be the actin-myosin force-generating machinery $(9,10)$. Myosin II is an actin-based motor protein that is important for cell migration through its effects on adhesion, lamellar protrusion, rear retraction, and polarity $(11,12)$. Using the actin network as a track, myosin II motors cross-link and contract the actin network, thus generating forces (13). Myosin II molecules are comprised of three pairs of peptides: two heavy chains of $230 \mathrm{kDa}$, two 20-kDa regulatory light chains (RLCs) that regulate NM II activity and two 17-kDa essential light chains (ELCs) that stabilize the heavy-chain structure. Isoforms of myosin II include myosin IIA, myosin IIB and myosin IIC, which contain heavy chains encoded by the genes MYH9, MYH10 and MYH14, respectively (14). Both myosin IIA and IIB are expressed in most mammalian cells, where they fulfill different but overlapping functions in cell migration. Myosin IIA localizes throughout the cell, even in protrusions. This protein plays a crucial role in rapid contractility, actin stress fiber formation, focal adhesion formation and cell-cell junctions (12). However, 
the function of myosin IIA in migration remains controversial. Several studies have indicated that myosin IIA deficiency leads to faster cell speeds relative to corresponding wild-type cells (12,15-17), while some reports argued that myosin IIA accelerate the migration speeds of breast cancer cells (18).

DT-13, a saponin of dwarf lilyturf tuber isolated from Ophiopogon japonicus (Thunb.), was found to display antitumor effects by inhibiting angiogenesis and proliferation through the regulation of VEGF and PI3K/Akt signaling, as well as anti-metastatic activity by inhibiting adhesion, migration and invasion via inhibition of MMP-2/9 or tissue factor $(19,20)$. We previously found that myosin IIA specifically responded to DT-13 via affinity chromatography (21); therefore, we hypothesized that myosin IIA may be a potential target of DT-13 to inhibit tumor metastasis in lung cancer. As hypoxia occurs during the deterioration of cancer, in this study, we used a hypoxic model to study the mechanisms underlying the antimetastatic activity of DT-13.

\section{Materials and methods}

Cell culture, transfection, and reagents. The cell line 95D was obtained from the Cell Bank of the Institute of Biochemistry and Cellular Biology, Chinese Academy of Sciences (Shanghai, China); 95D cells were maintained in RPMI-1640 medium (Gibco, Grand Island, NY, USA) supplemented with 10\% fetal bovine serum (FBS; Gibco), $80 \mathrm{U} / \mathrm{ml}$ penicillin, and $100 \mathrm{U} / \mathrm{ml}$ streptomycin. Cells were cultured in a humidified environment with $5 \% \mathrm{CO}_{2}$ at $37^{\circ} \mathrm{C}$. The shMYH9 and negative control shRNA were designed and synthesized by GenePharma (Shanghai, China). The shRNAs were transfected into 95D cells using lentivirus according to the manufacturer's instructions. DT-13 was a gift from Professor Bo-Yang Yu (China Pharmaceutical University, China). DT-13 was prepared at a stock concentration of $10 \mathrm{mM}$ in $100 \% \mathrm{DMSO}$, stored at $-20^{\circ} \mathrm{C}$, and diluted with phosphate-buffered solution (PBS) before each experiment. The final DMSO concentration did not exceed $0.1 \%$ throughout the study. The pharmacological inhibitor S(-)-blebbistatin (Merck) was used at a concentration of $100 \mu \mathrm{M}$. Phalloidin-FITC was obtained from Sigma. The following antibodies were used at the dilution rate of 1:1,000: ERK1/2, p-ERK1/2, c-Raf and p-c-Raf, $\beta$-actin, non-muscle myosin IIA, paxillin, p-paxillin (Tyr 118) were obtained from Cell Signaling Technology. Secondary anti-mouse IgG (Sigma) and anti-rabbit IgG (Cell Signaling Technology) were diluted at the ratio of 1:2,500. Immunoreactive proteins on membranes were visualized using ECL western blot detection reagents (Amersham, UK).

Cell spreading assays. Quantitative cell spreading assays were carried out in 96-well microtiter plates (Nunc, Naperville, IL, USA), which were coated with fibronectin (20 $\mu \mathrm{g} / \mathrm{ml}$; Sigma) for $1 \mathrm{~h}$ at room temperature and rinsed with PBS just before seeding the cells. Briefly, cells were collected from the culture plates using non-trypsin cell dissociation reagent and washed with PBS. Cell suspensions ( $200 \mu \mathrm{l} /$ well, $2 \times 10^{4}$ cells $\left./ \mathrm{ml}\right)$ with or without drugs were added to each well and incubated at $37^{\circ} \mathrm{C}$ for $30 \mathrm{~min}, 60$ and $120 \mathrm{~min}$.

Wound healing assay. 95D cells were grown to confluency in 24-well culture plates, after which a single linear wound was created through the monolayers using a sterile pipette tip. Monolayers were washed to remove cellular debris and placed in FBS-free media with DT-13 $(0.01,0.1$ and $1 \mu \mathrm{M})$, blebbistatin $(100 \mu \mathrm{M})$ or without any drugs. Sites at which wounds were to be measured were marked on the undersurface of the wells to ensure that measurements were taken at the same place (A defined area of the wound was photographed). Wounds were imaged at 0 and $12 \mathrm{~h}$ under phase-contrast microscopy.

Migration assay. The chemotactic motility of 95D cells was assayed using a Transwell chamber migration system (Millipore, USA) containing membranes with an $8-\mu \mathrm{m}$ pore size. $95 \mathrm{D}$ cell suspensions $\left(5.0 \times 10^{4}\right.$ cells per well $)$ were added to the upper chamber containing $1 \%$ FBS, while the lower chamber containing $10 \%$ FBS. DT-13 $(0.01,0.1$ and $1 \mu \mathrm{M})$ or blebbistatin $(100 \mu \mathrm{M})$ were added in the upper and lower chamber. After incubation for $12 \mathrm{~h}$ under normoxic or hypoxic condition, the upper surface of the membranes was swabbed to remove nonmigrated cells, and the cells attached onto the lower surface were fixed, stained, and counted microscopically. The migrated cells were quantified by manual counting, and five randomly chosen fields were analyzed for each group.

Invasion assay. Invasion assays were performed by using commercially available modified Boyden chambers (Millipore) layered with growth factor-reduced Matrigel (BD Biosciences), and chambers were incubated for $2 \mathrm{~h}$ at $37^{\circ} \mathrm{C}$. An equal number $\left(8 \times 10^{4}\right.$ cells) of $95 \mathrm{D}$ cells were suspended in the upper chamber [RPMI-1640 medium with $1 \%$ fetal bovine serum (FBS)], whereas medium containing $10 \%$ FBS was placed in the lower chamber. DT-13 $(0.01,0.1$ and $1 \mu \mathrm{M})$ or blebbistatin $(100 \mu \mathrm{M})$ were added in the upper and lower chambers. After 12-h incubation under normoxic or hypoxic condition, the cells on the upper surface of the membrane were completely removed. Then, the membrane was fixed with $4 \%$ paraformaldehyde for $10 \mathrm{~min}$ and stained with $0.1 \%$ crystal violet, and the upper-chamber cells were cleared using a cotton swab. Cells that invaded the Matrigel and reached the lower surface of the membrane in five different fields were counted and averaged for each group in a particular experiment. Each experiment was performed in triplicate.

Immunofluorescence staining. 95D cells were added into a 12 -well plate with $4 \times 10^{4}$ cells per well, cells were treated with DT-13 $(0.01,0.1$ and $1 \mu \mathrm{M})$ or blebbistatin $(100 \mu \mathrm{M})$ under normoxic and hypoxic conditions for $12 \mathrm{~h}$, washed twice with PBS, and fixed with $4 \%$ paraformaldehyde for $30 \mathrm{~min}$. The fixed cells were permeabilised with $0.1 \%$ Triton X-100 for $10 \mathrm{~min}$ at room temperature, washed with TBST and blocked with blocking buffer $(5 \% \mathrm{BSA})$ at $37^{\circ} \mathrm{C}$ for $1 \mathrm{~h}$ and then incubated with primary antibody (diluted to 1:300 in blocking buffer) at $37^{\circ} \mathrm{C}$ for $1 \mathrm{~h}$, followed by Alexa-conjugated secondary antibodies diluted to 1,000-fold in blocking buffer and Hoechst 33342 (Beyotime) for $1 \mathrm{~h}$ at room temperature in the dark. The cells were washed three times with TBST before imaging on a confocal microscope.

Western blot analysis. 95D cells were added into the 6-well plate with $4 \times 10^{5}$ cells per well, cells were treated with different concentrations $(0.01,0.1$ and $1 \mu \mathrm{M})$ of DT-13 after adhered 
A
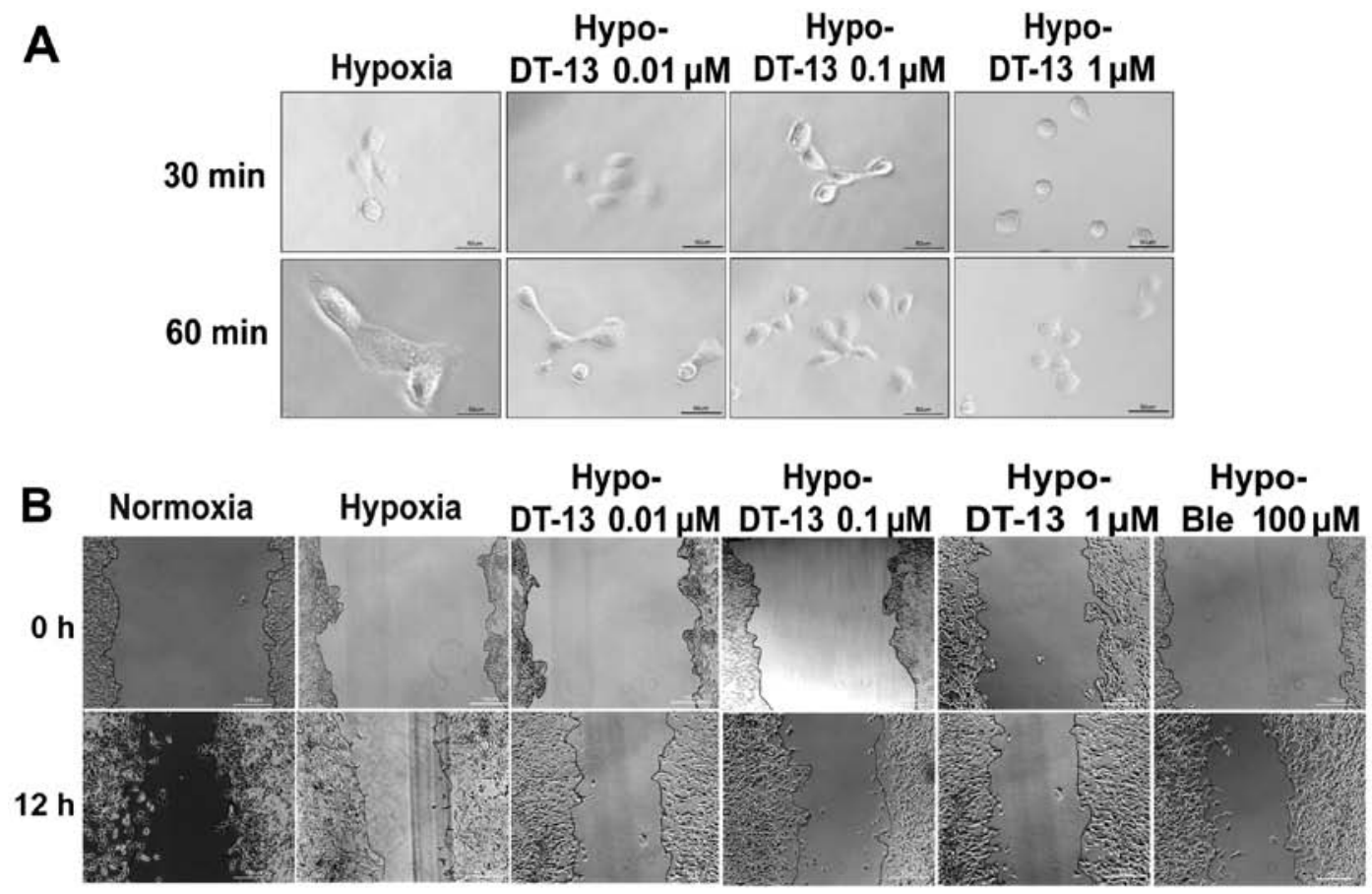

C

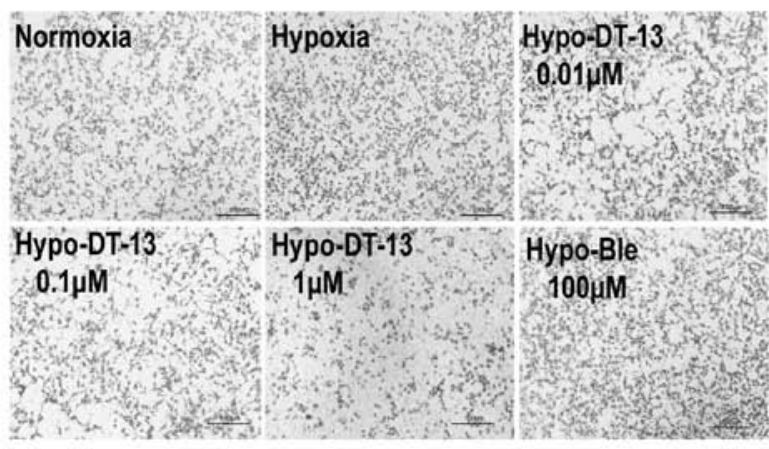

D

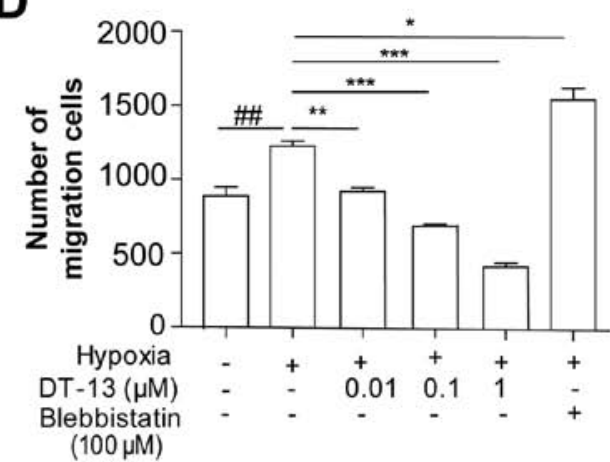

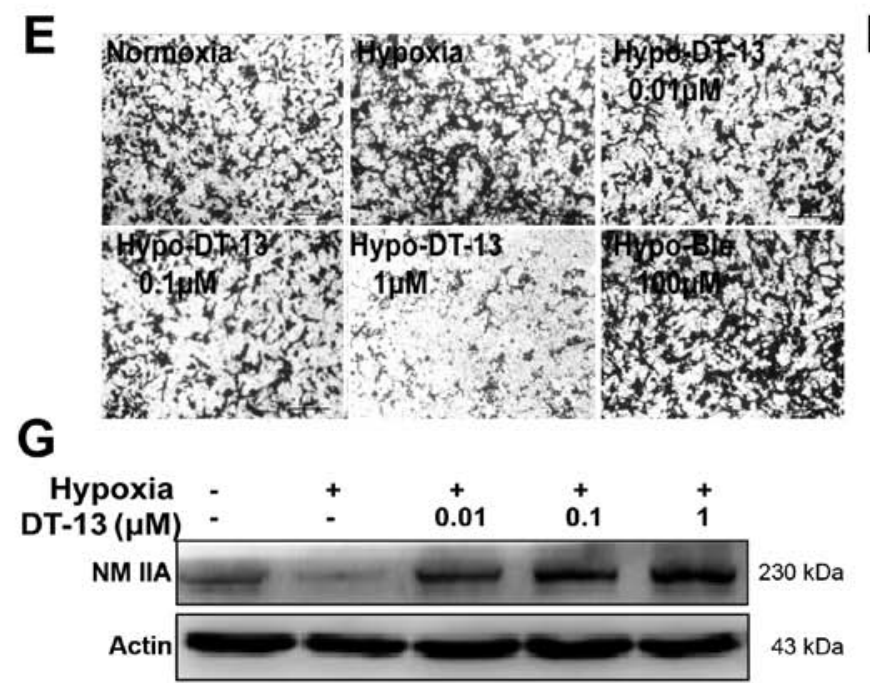

$\mathbf{F}$

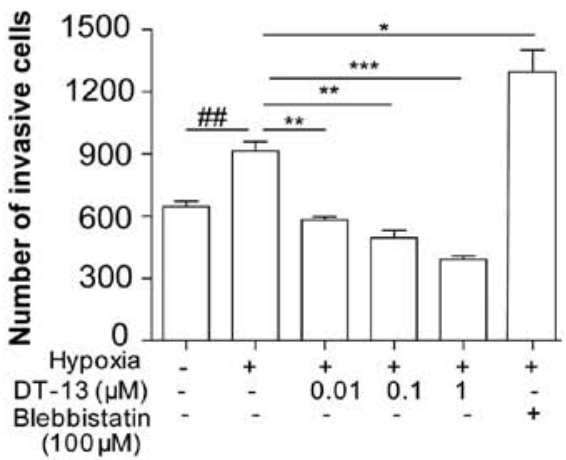

Figure 1. Inhibitory effects of DT-13 on 95D cell migration and invasion under hypoxia. (A) Spreading assays were used to examine the spreading ability of 95D cells treated with $0.01,0.1$ and $1 \mu \mathrm{M}$ DT-13 under hypoxia; images were taken at 30 and 60 min after drug treatment. (B) Representative images of cells were taken at 0 and $12 \mathrm{~h}$ after wounding, and cells were treated with $0.01,0.1$ or $1 \mu \mathrm{M}$ of DT-13 and $100 \mu \mathrm{M}$ blebbistatin in serum-free medium for $12 \mathrm{~h}$ (magnification, x100). (C) Effects of DT-13 on 95D cell migration. In the Transwell cell migration assay, cells were seeded in the upper Transwell chamber and treated with DT-13 $(0.01,0.1$ and $1 \mu \mathrm{M})$ and blebbistatin $(100 \mu \mathrm{M})$ for $12 \mathrm{~h}$ in serum-free medium. The lower chamber contained medium supplemented with $10 \%$ FBS. The downward side of the membrane was stained with crystal violet and Eosin Y and counted by an inverted microscope. (D) Statistical analysis of 95D cell migration counted in 5 random fields after Transwell migration. (E) Effects of DT-13 on 95D cell invasion. In the Transwell cell invasion assay, cells were seeded in the upper portion of Transwell chamber coated with Matrigel and treated with DT-13 $(0.01,0.1$ and $1 \mu \mathrm{M})$ and blebbistatin $(100 \mu \mathrm{M})$ for $12 \mathrm{~h}$ in serum-free medium; medium containing 10\% FBS was placed in the lower chamber (magnification, $\mathrm{x} 100$ ). After $12 \mathrm{~h}$ of incubation, the downward side of the membrane was stained and counted on an inverted microscope. (F) Statistical analysis of $95 \mathrm{D}$ cell Matrigel invasion counted in 5 random fields. ${ }^{\#} \mathrm{P}<0.05$, ${ }^{\# \#} \mathrm{P}<0.01,{ }^{\# \# \#} \mathrm{P}<0.001$ versus normoxia; ${ }^{*} \mathrm{P}<0.05,{ }^{* *} \mathrm{P}<0.01,{ }^{* * *} \mathrm{P}<0.001$ versus hypoxia. (G) Western blot analysis of NMIIA expression in $95 \mathrm{D}$ cells after treated with DT-13 $(0.01,0.1$ and $1 \mu \mathrm{M})$ for $12 \mathrm{~h}$. 
and then exposed to normoxia or hypoxia for $12 \mathrm{~h}$. Cellular protein extraction and western blot analysis were performed as previously described. Total proteins $(40 \mu \mathrm{g})$ were separated via $10 \%$ SDS-PAGE and then electroblotted onto polyvinylidene difluoride membranes (Millipore). After blocking with $5 \%$ BSA, $0.1 \%$ Tween-20 in TBST at room temperature for $1 \mathrm{~h}$, the blots were incubated overnight with primary antibodies at $4^{\circ} \mathrm{C}$. The blots were subsequently washed and incubated with secondary antibodies for $1 \mathrm{~h}$ at room temperature. Proteins were detected by enhanced chemiluminescence using the ECL substrate (Amersham Pharmacia Biotech).

Orthotopic implantation nude mouse model. Six-week-old, female nude mice were used for orthotopic implantation. 95D cells were suspended in 50\% Matrigel (BD) in PBS. A sample of $100 \mu \mathrm{l}$ of $1 \times 10^{6}$ cells/mouse was injected into the right mouse lung to generate tumors in a natural orthotopic site. One week later, the mice were intra-gastrically administered with DT-13 (2.5, 5 and $10 \mathrm{mg} / \mathrm{kg} / 1$ body weight, daily) in $0.5 \%$ CMC-Na solution or with topotecan hydrochloride which used as positive drug $(2 \mathrm{mg} / \mathrm{kg} / 1$ body weight) twice a week via tail-vein injection ( 8 mice per group). Mice in the control group were intra-gastrically administered $0.5 \% \mathrm{CMC}-\mathrm{Na}$ solution daily and this in vivo experiment was finished after the mice in control group all died. Ethics approval was received from the Committee of the Southeastern University.

Statistical analysis. The data represent at least three independent experiments and are expressed as the means \pm SEM. For statistical analysis, One-way ANOVA or Student's t-test was used when appropriate.

\section{Results}

DT-13 inhibits the effects of hypoxia on 95D cell migration and invasion. Lamellipodia formation is required for cancer cell migration. To verify whether DT-13 suppresses lamellipodia formation in lung cancer $95 \mathrm{D}$ cells, cells were treated with DT-13 $(0.01,0.1$ and $1 \mu \mathrm{M})$ for $12 \mathrm{~h}$ and then plated onto a fibronectin-coated 96-well plate. After adhering to the fibronectin, we found that cells without DT-13 treatment showed rapid, time-dependent lamellipodia formation, whereas this extension was slower in the groups treated with DT-13 $(0.01$, 0.1 and $1 \mu \mathrm{M})$. These results suggested that DT-13 blocked the spreading of 95D cells on fibronectin, as shown in Fig. 1A.

Next, scratch migration assays and Transwell migration assays were performed to evaluate the effects of DT- 13 on cell migration under hypoxia. We found that hypoxic conditions enhanced the migration capacity of 95D cells compared with normoxia; however, different concentrations of DT-13 noticeably decreased tumor cell migration under hypoxia in a dose-dependent manner (Fig. 1B, C and E). The acquisition of an invasive phenotype of cancer cells is a critical step for tumor progression. Matrigel-coated filters are widely used to examine invasive migration through a three-dimensional extracellular matrix. We found that hypoxia was necessary to accelerate the invasion of 95D cells compared with normoxia; however, after treatment of DT-13, we found that this cell invasion activity was effectively decreased under hypoxia in a dose-dependent manner (Fig. 1D and F).
A

B
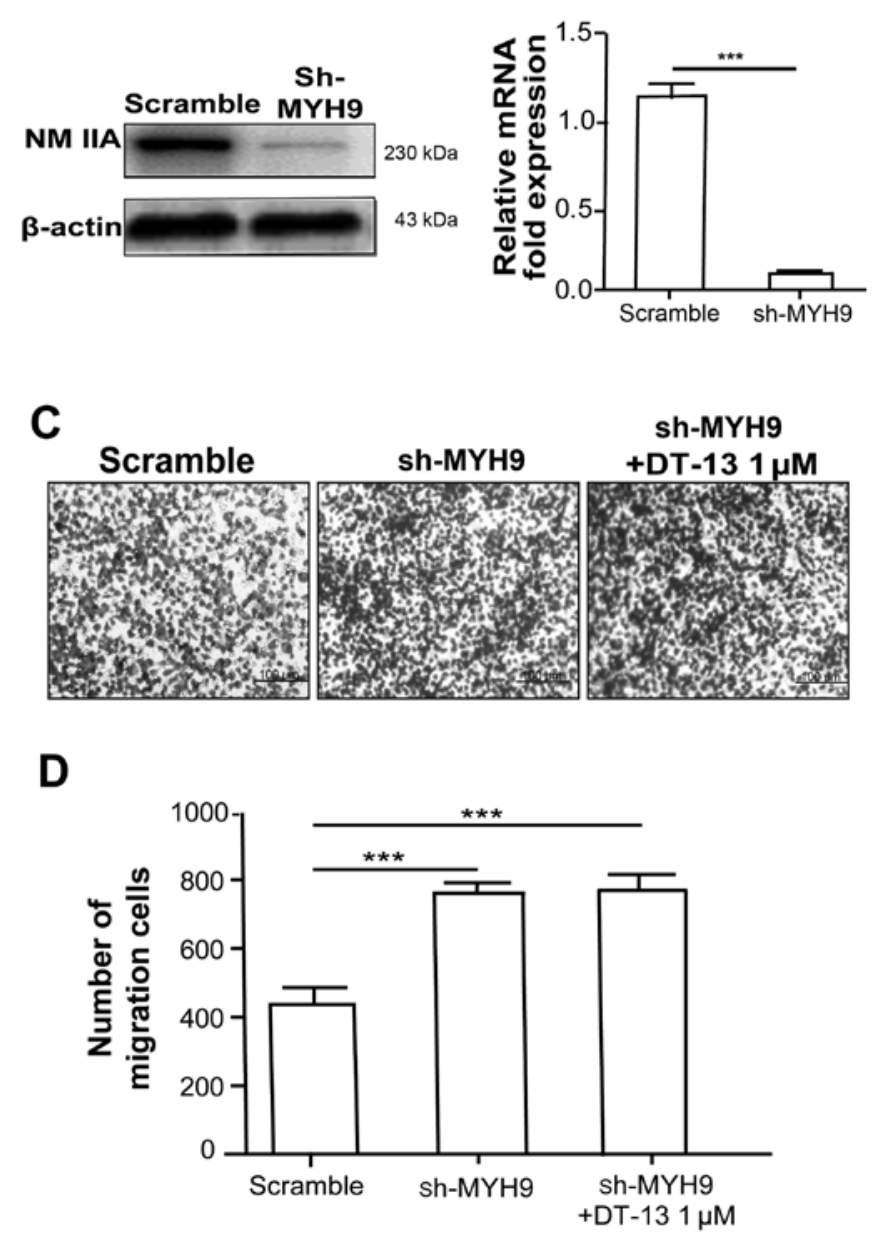

Figure 2. NMIIA is required for the inhibitory effect of DT-13 on cancer cell metastasis. (A) Western blot analysis of NMIIA expression after MYH9 knockdown. (B) qPCR analysis of MYH9 expression after MYH9 knockdown. (C) Effect of DT-13 on the migration of lung cancer cells transfected with MYH9 shRNA. 95D cell suspensions were added to the upper chamber containing $1 \%$ FBS, while the lower chamber containing $10 \%$ FBS. DT-13 $1 \mu \mathrm{M}$ were added in the upper and lower chambers. After incubation for $12 \mathrm{~h}$ under hypoxic condition, (D) Statistical analysis of 95D cell migration counted in 5 random fields after a Transwell assay. ${ }^{* * * *} \mathrm{P}<0.001$ versus sh-scramble cells (magnification, $\mathrm{x} 100$ ).

NMIIA, a conventional motor protein known to generate intracellular contractile force and tension by associating with F-actin, has been implicated in the regulation of many cellular processes, including cell spreading, migration and cytokinesis $(12,17)$. Our previous study showed that DT-13 interacted specifically with NMIIA based on affinity chromatography analysis (21), which led us to hypothesize that NMIIA may be important for the anti-metastatic effect of DT-13. Interestingly, we found that hypoxia led to the downregulation of NMIIA, while DT-13 could upregulate NMIIA under hypoxia (Fig. 1G). Furthermore, to verify the effect of NMIIA on 95D cells migration and invasion in depth, $100 \mu \mathrm{M}$ blebbistatin was used as a pharmacological inhibitor of myosin II, and we found that both the migration and invasion of 95D cells were enhanced by treatment with blebbistatin (Fig. 1B, C and E). These data indicated that the inhibition of NMII accelerated the migration and invasion of 95D cells, which was in agreement with previous studies $(16,22)$. 


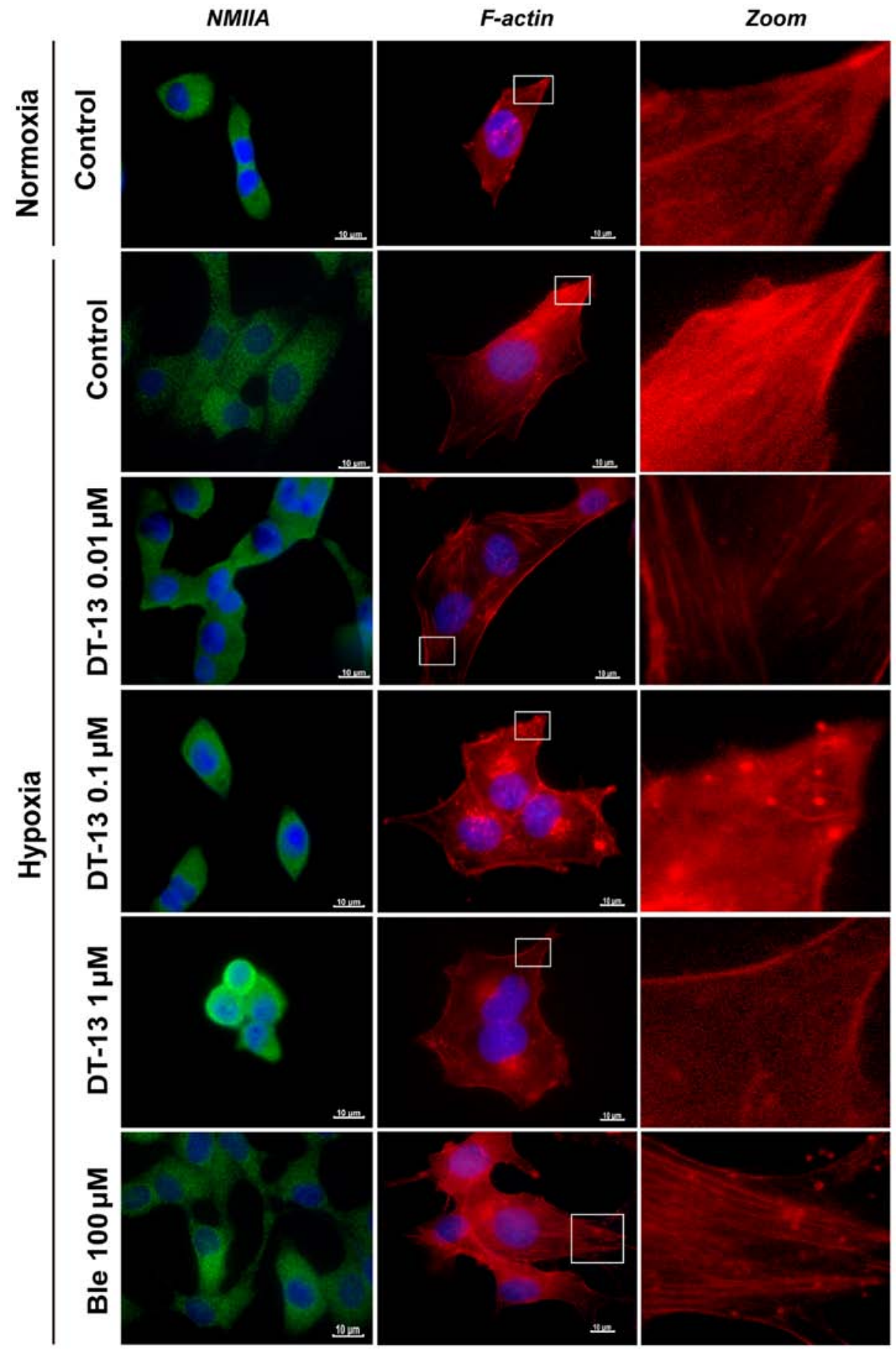

Figure 3. DT-13 attenuates migration by redistributing NMIIA and rearrangement of actin cytoskeletal in 95D cells under hypoxia. Immunofluorescence staining of NMIIA antibody (green), F-actin (red) and Hoechst (blue) was used to stain the nucleus. 95D cells were treated with PBS, $0.01,0.1$ or $1 \mu$ M DT- 13 and $100 \mu \mathrm{M}$ blebbistatin under normoxic and hypoxic conditions for $12 \mathrm{~h}$ (magnification for NMIIA, x400; for F-actin, x600).

NMIIA is required for the inhibitory effect of DT-13 on cancer cell metastasis. To verify whether NMIIA is responsible for the inhibitory effect of DT-13 on cancer metastasis, the expression of NMIIA in 95D cells was depleted by MYH9-targeted shRNA (Fig. 2A and B). Cells transfected with MYH9 shRNA significantly increased migration compared with sh-scramble cells under hypoxia (Fig. 2C and D). To further examine whether DT-13 inhibits 95D cell migration through the motor activity of myosin IIA, shMYH9 cells were treated with DT-13 $(1 \mu \mathrm{M})$ in Transwell migration assays. Our results showed that the inhibitory effect of DT-13 on cell migration was abolished in shMYH9 cells. Together, these results further indicated that the anti-migration effect of DT-13 occured through the upregulation of NMIIA.

DT-13 attenuates migration by redistributing NMIIA in 95D cells under hypoxia. NMIIA regulates the tension and contractility of actin cytoskeleton. Previous studies (14) indicated that myosin IIA exerts a retraction force to draw the cell cytoplasm closer to the nucleus and attenuate cell movement. To explore whether DT-13 alters the distribution of NMIIA in 95D cells, we used immunofluorescence analysis to study the distribution 


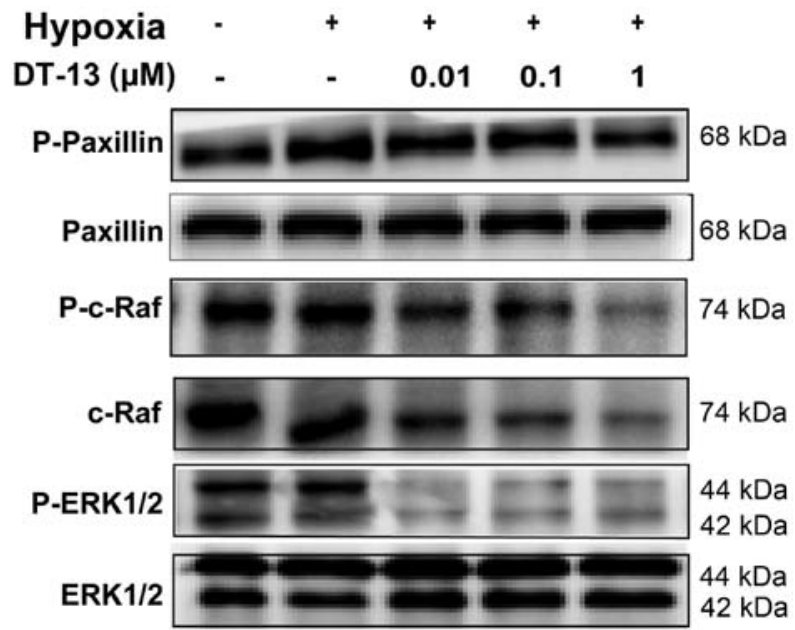

Figure 4. DT-13 inhibits 95D cell migration and invasion via repression of the Raf-ERK1/2 signaling pathway. Using specific antibodies to analyze the expression of paxillin, p-paxillin, p-c-Raf, total c-Raf, p-ERK1/2, total ERK $1 / 2$ and $\beta$-actin in 95D cells after treated with DT-13 (0.01, 0.1 and $1 \mu \mathrm{M})$ for $12 \mathrm{~h}$ of NMIIA. Compared with normoxia, we observed a morphological reorganization of 95D cells and redistribution of NMIIA under hypoxic conditions, with NMIIA predominantly localizing to the cell periphery. Nevertheless, we observed that NMIIA was expressed in the central region and predominantly accumulated in the nuclear periphery after treatment with DT-13. Thus, we hypothesize that DT-13 may promote NMIIA accumulation in the peri-nuclear area to inhibit cell migration. To verify this hypothesis, we treated 95D cells with blebbistatin $(100 \mu \mathrm{M})$ under hypoxia and observed the redistribution of NMIIA to the cell periphery (Fig. 3). Collectively, these observations suggested that DT-13 affected the distribution of NMIIA and thus has a major role in the inhibition of 95D cell migration.

DT-13 regulates actin cytoskeletal rearrangement in $95 D$ cells. The dynamic regulation of the filamentous actin (F-actin) cytoskeleton is crucial for cell migration, and myosin IIA is involved in the formation of actin stress fibers. Therefore, we analyzed whether DT-13 alters cellular F-actin arrangement under hypoxia.
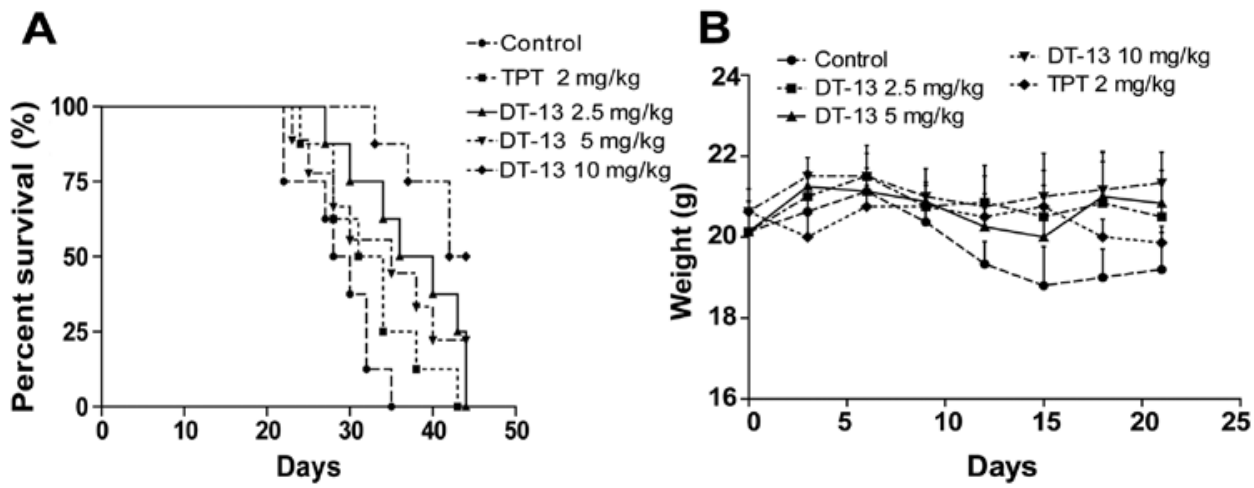

C
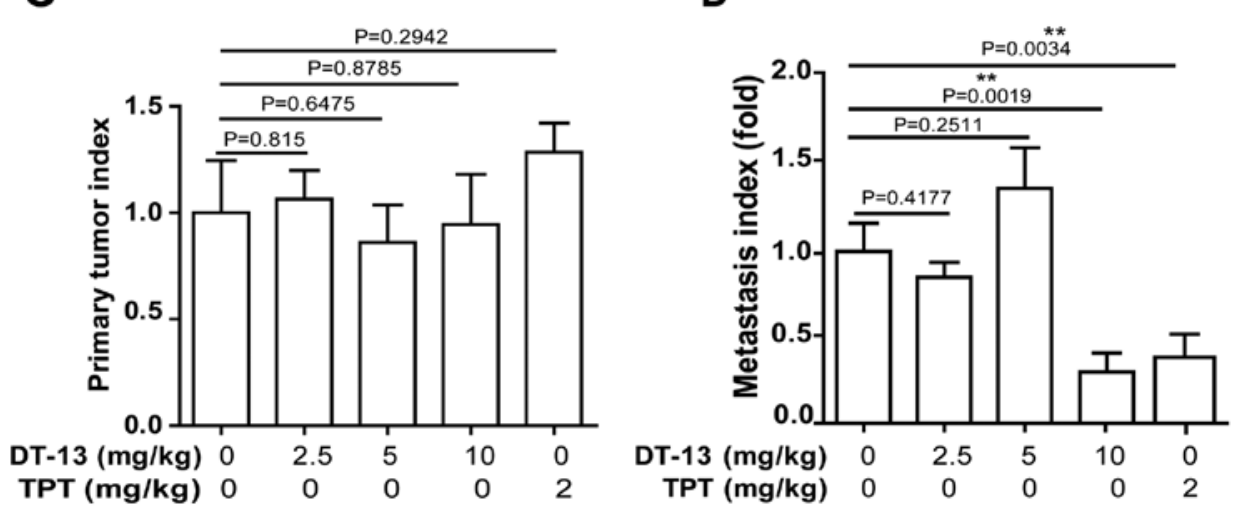

E

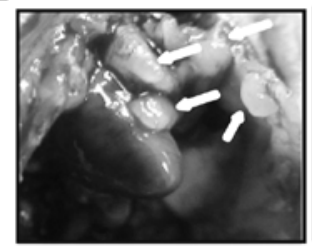

Control

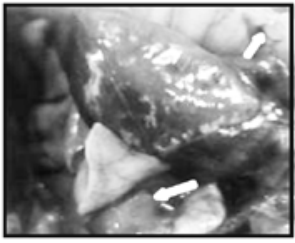

DT-13 $2.5 \mathrm{mg} / \mathrm{kg}$

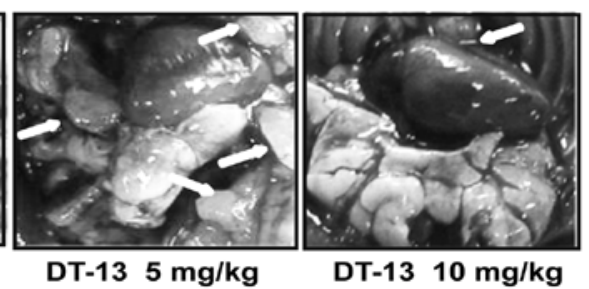

Figure 5. DT-13 inhibits human lung cancer 95D cell metastasis in vivo. (A) DT-13 administration prolongs the survival of mice bearing orthotopic human NSCLC 95D cell xenografts. (B) DT-13 stabilizes the weights of mice bearing orthotopic human NSCLC 95D cell xenografts. (C) DT-13 slightly inhibits the primary tumor growth. The lung primary tumor index was calculated using the orthotopic lung weight/body weight ratio. (D) DT-13 significantly inhibited tumor metastasis. The lung metastasis tumor index was calculated using the ratio of metastasis lung weight/body weight. (E) Images of lungs were taken using a digital camera. 
First, we compared the formation of F-actin in 95D cells in two different environments (normoxia and hypoxia) for $12 \mathrm{~h}$ and observed cytoskeletal variations by staining for F-actin with phalloidin-FITC. We found that hypoxic environments stimulated the remodeling of actin filaments and also changed the cellular morphology; moreover, the formation of F-actin in $95 \mathrm{D}$ cells was accelerated under hypoxia compared to normoxia. However, after treatment with DT-13, we observed a noticeable disruption of actin stress fibers and loss of actin stress fibers in 95D cells (Fig. 3). In contrast to this observation, treatment with blebbistatin $(100 \mu \mathrm{M})$ instead of DT-13 for $12 \mathrm{~h}$ had the opposite effect, resulting in a significant increase in the number of stress fibers with polymerization at the leading edge. These findings indicated that DT-13 may inhibit cell migration through reducing the formation of F-actin in 95D cells.

DT-13 inhibits $95 D$ cell migration and invasion via repression of the Raf-ERK1/2 signaling pathway. Given that DT-13 could impair 95D cell migration and invasion by regulating NMIIA, we next investigated the potential mechanisms involved. As NMIIA associates with FA proteins, we explored whether the expression of FA proteins and the related adhesion signaling pathway was modulated.

Thus, immunoblot analysis was performed to examine a possible effect of DT-13 on p-paxillin and paxillin under hypoxic condition, which have been shown to be downstream effectors of NMIIA. Our results showed that the level of p-paxillin was upregulated under hypoxia, while DT-13 reversed the hypoxic effect (Fig. 4).

Multiple NMIIA downstream effectors have been shown to mediate the migration behavior of epithelial cells by activating the Raf-ERK1/2 signaling cascade (25). To assess whether DT-13 increased the expression of NMIIA under hypoxia via Raf-ERK1/2 signaling, we conducted immunoblot analysis and showed an association between DT-13 and the Raf-ERK1/2 pathway. As p-c-Raf and p-ERK1/2 were significantly downregulated after treatment with DT-13 in 95D cells under hypoxia, while total c-Raf levels were also downregulated (Fig. 4). Cumulatively, these observations suggested that Raf-ERK1/2 inhibition was responsible for the suppression of migration and invasion by DT-13 treatment in 95D cells.

DT-13 inhibits human lung cancer 95D cell metastasis in vivo. To further evaluate the efficacy of DT-13 in a clinically relevant non-small cell lung cancer (NSCLC) model, Matrigel-embedded 95D cells were implanted into mouse lungs at a natural orthotopic site that provides a biologically appropriate environment for tumor growth, invasion and metastasis. The survival rate was significantly increased after treatment with DT-13 $(10 \mathrm{mg} / \mathrm{kg})(\mathrm{P}=0.0014)$ and DT-13 $(2.5 \mathrm{mg} / \mathrm{kg})(\mathrm{P}=0.0148)$ compared with control group (Fig. 5A). Then, we observed that the weight of the mice in the group without DT-13 treatment gradually decreased compared to the DT-13-treated group (Fig. 5B). Further analysis of the orthotopic lung-weight/mouse-weight ratio showed that DT-13 had no effect on tumor growth of the primary cancer (Fig. 5C). However, the metastasis index indicated that $10 \mathrm{mg} / \mathrm{kg}$ DT-13 treatment significantly impeded the metastatic spread of cancer to other lung areas $(P=0.0019)$ and that $2.5 \mathrm{mg} / \mathrm{kg}$ DT-13 treatment slightly inhibited metastasis $(\mathrm{P}=0.4177)$, while the medium dose exhibited pro-metastatic effect but without statistical difference (Fig. 5D). In addition, we also found that tumor metastasis along the parietal peritoneum in DT-13 treated groups was significantly decreased compared with the control group (Fig. 5E).

\section{Discussion}

In this study, we first showed that hypoxia leads to the redistribution of NMIIA to the cell periphery and strongly downregulates NMIIA protein expression in 95D cells compared with normoxia. Furthermore, we found the Raf-ERK1/2 signaling is involved in regulating NMIIA and its downstream molecular paxillin under hypoxia. Moreover, DT-13 reverses the hypoxic effect via greatly inhibiting Raf-ERK1/2 signaling and further regulating NMIIA.

According to previous studies, both NMIIA and NMIIB can bind and contract actin filaments to generate force. However, NMIIA and NMIIB have different cellular localizations, which lead to different functions. NMIIA primarily localizes to the front, protruding edge and is required for adhesion maturation. NMIIA is also required for inward cellular contractility, the formation of actin stress fibers, and morphogenic cell clustering (23). Controversially, previous research suggested that NMIIA deficiency leads to faster cell migration in multiple cell types $(18,22)$, whereas other studies argued that myosin IIA overexpression is implicated in enhanced cancer cell migration and metastasis (24-26). However, this controversy is lessened by recent reports on the roles of myosin IIA in the posttranscriptional stabilization of p53 activity and the repression of squamous cell carcinoma in mice (22).

It has been reported that the absence of actin stress fibers in NMIIA deficient cells may increase the concentration of cytoplasmic actin monomers, which in turn promotes actin polymerization at the leading edge, and NMIIA-deficient cells have fewer cell-extracellular matrix adhesions, thus requiring less contractile force to achieve de-adhesion, which is an essential step in cell migration (27). The present study showed that hypoxia downregulates NMIIA expression and induces the redistribution of NMIIA to the cellular peripheries thus possibly weakening their anchoring force, and this further accelerates the formation of F-actin in 95D cells. Our results showed that DT-13 reverses the effect of hypoxia by lessening the formation of F-actin. Moreover, we found that DT-13 inhibits human lung cancer 95D cells spreading on fibronectin or migration and invasion in vitro, and these findings are consistent with orthotopic lung cancer metastasis in vivo. Our findings strongly support the notion that DT-13 upregulates NMIIA and disrupts actin stress fibers, which are key factors associated with cell migration. Firthermore, we also verified these findings by knocking down MYH9 in 95D cells or using blebbistain to inhibit NMIIA, which all accelerate cell migration (Fig. 2C and D).

Paxillin is a component of focal adhesion complexes, which mediates focal adhesion assembly and cell motility (28). In this study, we found that hypoxia activates p-paxillin, while DT-13 downregulates the expression of p-paxillin. This effect may be the result of DT-13-induced NMIIA upregulation, while NMIIA may increase the anchoring forces generated through NMIIA to FAS. Furthermore, we discovered that the Raf-ERK1/2 signaling pathway is the major pathway through 
which DT-13 exerts the cell migration-inhibitory effect. On the one hand, upregulation of NMIIA expression by DT-13 treatment induces significant inactivation of ERK by decreasing phospho-ERK1/2 levels and inhibits c-Raf kinase under hypoxia. On the other hand, as ERK1/2 is known to complex and colocalize with paxillin (13), DT-13 downregulates paxillin and ERK1/2 may inhibit the binding of ERK1/2 to paxillin, and further inhibit the migration of $95 \mathrm{D}$ cells, but this hypothesis still need to be confirmed. Besides paxillin, previous studies reported that calpain and MMPs are involved in the Raf-ERK1/2 signaling pathway regulating NMIIA (29), and our previous results showed that DT-13 could repress the metastasis of human lung cancer A549 cells by inhibiting MMPs (20). Therefore, we speculate the MMPs are involved in the anti-metastatic effect of DT-13 in 95D cells.

Thus, it is necessary to clarify how DT-13 suppresses 95D cell metastasis by regulating NMIIA under hypoxia. Hypoxia is a potent inducer of HIF-1 $\alpha$ expression, and HIF-1 $\alpha$ plays a critical role in tumor progression and dissemination. Our previous report showed DT-13 could inhibit the expression of HIF-1 $\alpha$, thus we hypothesize DT-13 may indirectly regulate NMIIA by the inhibition of HIF-1 $\alpha$. In this study, we showed hypoxia redistributes NMIIA to the cell periphery, which reduces the adhesion effect, while NMIIA accumulated in the nuclear periphery after treated with DT-13. Therefore, these results demonstrate that DT-13 may directly regulate NMIIA under hypoxic condition.

In conclusion, this study demonstrated, hypoxia promotes the migratory potential of in vitro cultured 95D cells by downregulating NMIIA expression and redistributing NMIIA to the periphery of 95D cells via rearrangement of F-actin and activation of Raf-ERK1/2 signaling pathway. The experiments further indicated the potential of DT-13 to hamper metastasis in vitro and in vivo, acting through upregulation of NMIIA and redistribution NMIIA to the nuclear periphery, further disrupting actin stress fibers and inhibiting the Ras-ERK1/2 signaling pathway. This conclusion is also supported by MYH9 knockdown cells or blebbistatin treated 95D cells. Thus, NMIIA may play an important role in the anti-metastatic process of DT-13. However, further studies are required to determine whether NMIIB, MMPs and calpain are involved in the anti-metastatic effect of DT-13, and more in-depth experiments, such as changes of NMIIA in vivo after treatment with DT-13, and clinic trials also need to be performed. In general, this study provides the first demonstration that DT-13 inhibits 95D cell migration and invasion through the activation of NMIIA via inhibition of Ras-ERK1/2 signaling pathway. Therefore, our findings offer new insights into the potential significance of DT-13 in lung cancer.

\section{Acknowledgements}

This study was supported by the National Natural Science Fund nos. 81302794, 81102853 and 81573456.

\section{References}

1. Edwards BK, Noone AM, Mariotto AB, Simard EP, Boscoe FP, Henley SJ, Jemal A, Cho H, Anderson RN, Kohler BA, et al: Annual Report to the Nation on the status of cancer, 1975-2010, featuring prevalence of comorbidity and impact on survival among persons with lung, colorectal, breast, or prostate cancer. Cancer 120: 1290-1314, 2014.
2. Wang Y, Liu Y, Malek SN, Zheng P and Liu Y: Targeting HIF1 $\alpha$ eliminates cancer stem cells in hematological malignancies. Cell Stem Cell 8: 399-411, 2011.

3. Luo W, Hu H, Chang R, Zhong J, Knabel M, O'Meally R, Cole RN, Pandey A and Semenza GL: Pyruvate kinase M2 is a PHD3-stimulated coactivator for hypoxia-inducible factor 1. Cell 145: 732-744, 2011

4. Mak P, Leav I, Pursell B, Bae D, Yang X, Taglienti CA, Gouvin LM, Sharma VM and Mercurio AM: ERbeta impedes prostate cancer EMT by destabilizing HIF-lalpha and inhibiting VEGF-mediated snail nuclear localization: Implications for Gleason grading. Cancer Cell 17: 319-332, 2010.

5. Swietach P, Vaughan-Jones RD and Harris AL: Regulation of tumor $\mathrm{pH}$ and the role of carbonic anhydrase 9. Cancer Metastasis Rev 26: 299-310, 2007.

6. Chan DA and Giaccia AJ: Hypoxia, gene expression, and metastasis. Cancer Metastasis Rev 26: 333-339, 2007.

7. Semenza GL: Hypoxia-inducible factors in physiology and medicine. Cell 148: 399-408, 2012.

8. Zhang H, Wong CCL, Wei H, Gilkes DM, Korangath P, Chaturvedi P, Schito L, Chen J, Krishnamachary B, Winnard PT Jr, et al: HIF-1-dependent expression of angiopoietin-like 4 and L1CAM mediates vascular metastasis of hypoxic breast cancer cells to the lungs. Oncogene 31: 1757-1770, 2012.

9. Estecha A, Sánchez-Martín L, Puig-Kröger A, Bartolomé RA, Teixidó J, Samaniego R and Sánchez-Mateos P: Moesin orchestrates cortical polarity of melanoma tumour cells to initiate $3 \mathrm{D}$ invasion. J Cell Sci 122: 3492-3501, 2009.

10. Poincloux R, Collin O, Lizárraga F, Romao M, Debray M, Piel M and Chavrier P: Contractility of the cell rear drives invasion of breast tumor cells in 3D Matrigel. Proc Natl Acad Sci USA 108: 1943-1948, 2011.

11. Dahan I, Yearim A, Touboul Y and Ravid S: The tumor suppressor Lgl1 regulates NMII-A cellular distribution and focal adhesion morphology to optimize cell migration. Mol Biol Cell 23: 591-601, 2012.

12. Vicente-Manzanares M, Ma X, Adelstein RS and Horwitz AR: Non-muscle myosin II takes centre stage in cell adhesion and migration. Nat Rev Mol Cell Biol 10: 778-790, 2009.

13. Clark K, Langeslag M, Figdor CG and van Leeuwen FN: Myosin II and mechanotransduction: A balancing act. Trends Cell Biol 17: 178-186, 2007.

14. Betapudi V: Myosin II motor proteins with different functions determine the fate of lamellipodia extension during cell spreading. PLoS One 5: e8560, 2010.

15. Doyle AD, Kutys ML, Conti MA, Matsumoto K, Adelstein RS and Yamada KM: Micro-environmental control of cell migration - myosin IIA is required for efficient migration in fibrillar environments through control of cell adhesion dynamics. J Cell Sci 125: 2244-2256, 2012.

16. Liu Z, van Grunsven LA, Van Rossen E, Schroyen B, Timmermans JP, Geerts A and Reynaert H: Blebbistatin inhibits contraction and accelerates migration in mouse hepatic stellate cells. Br J Pharmacol 159: 304-315, 2010.

17. Liu Z, Van Rossen E, Timmermans JP, Geerts A, van Grunsven LA and Reynaert H: Distinct roles for non-muscle myosin II isoforms in mouse hepatic stellate cells. J Hepatol 54: 132-141, 2011.

18. Betapudi V, Licate LS and Egelhoff TT: Distinct roles of nonmuscle myosin II isoforms in the regulation of MDA-MB-231 breast cancer cell spreading and migration. Cancer Res 66: 4725-4733, 2006.

19. Zhao R, Sun L, Lin S, Bai X, Yu B, Yuan S and Zhang L: The saponin monomer of dwarf lilyturf tuber, DT-13, inhibits angiogenesis under hypoxia and normoxia via multi-targeting activity. Oncol Rep 29: 1379-1386, 2013.

20. Zhang YY, Liu JH, Kou JP, Yu J and Yu BY: DT-13, a steroidal saponin from Liriope muscari L. H. Bailey, suppresses A549 cells adhesion and invasion by inhibiting MMP-2/9. Chin J Nat Med 10: 436-440, 2012.

21. Yu XW, Lin S, Du HZ, Zhao RP, Feng SY, Yu BY, Zhang LY, Li RM, Qian CM, Luo XJ, et al: Synergistic combination of DT-13 and topotecan inhibits human gastric cancer via myosin IIA-induced endocytosis of egf receptor in vitro and in vivo. Oncotarget doi: 10.18632, 2016.

22. Schramek D, Sendoel A, Segal JP, Beronja S, Heller E, Oristian D, Reva B and Fuchs E: Direct in vivo RNAi screen unveils myosin IIa as a tumor suppressor of squamous cell carcinomas. Science 343: 309-313, 2014

23. Liu Z, Ho CH and Grinnell F: The different roles of myosin IIA and myosin IIB in contraction of 3D collagen matrices by human fibroblasts. Exp Cell Res 326: 295-306, 2014. 
24. Casalou C, Seixas C, Portelinha A, Pintado P, Barros M, Ramalho JS, Lopes SS and Barral DC: Arl13b and the non-muscle myosin heavy chain IIA are required for circular dorsal ruffle formation and cell migration. J Cell Sci 127: 2709-2722, 2014.

25. Derycke L, Stove C, Vercoutter-Edouart AS, De Wever O, Dollé L, Colpaert N, Depypere H, Michalski JC and Bracke M: The role of non-muscle myosin IIA in aggregation and invasion of human MCF-7 breast cancer cells. Int J Dev Biol 55: 835-840, 2011.

26. Xia ZK, Yuan YC, Yin N, Yin BL, Tan ZP and Hu YR: Nonmuscle myosin IIA is associated with poor prognosis of esophageal squamous cancer. Dis Esophagus 25: 427-436, 2012.
27. Jorrisch MH, Shih W and Yamada S: Myosin IIA deficient cells migrate efficiently despite reduced traction forces at cell periphery. Biol Open 2: 368-372, 2013.

28. Zhang H, Chen Y, Wadham C, McCaughan GW, Keane FM and Gorrell MD: Dipeptidyl peptidase 9 subcellular localization and a role in cell adhesion involving focal adhesion kinase and paxillin. Biochim Biophys Acta 1853: 470-480, 2015.

29. Babbin BA, Koch S, Bachar M, Conti MA, Parkos CA, Adelstein RS, Nusrat A and Ivanov AI: Non-muscle myosin IIA differentially regulates intestinal epithelial cell restitution and matrix invasion. Am J Pathol 174: 436-448, 2009. 